\title{
ACEITE ESENCIAL DE Eucalyptus globulus Labill Y Eucalyptus nitens H. Deane \& Maiden (MYRTACEAE) PARA EL CONTROL DE Sitophilus zeamais Motschulsky
}

\author{
ESSENTIAL OIL OF Eucalyptus globulus Labill AND Eucalyptus nitens H. \\ Deane \& Maiden (MYRTACEAE) FOR THE CONTROL OF Sitophilus zeamais \\ Motschulsky
}

Raúl González-Guiñez ${ }^{1}$, Gonzalo Silva-Aguayo ${ }^{1 *}$, Angélica Urbina-Parra ${ }^{1}$, y Macarena GerdingGonzález $^{1}$

${ }^{1}$ Departamento de Producción Vegetal, Facultad de Agronomía, Universidad de Concepción, Avenida Vicente Méndez 595, Casilla 537, Chillán. Chile.

* Autor para correspondencia E-mail: gosilva@udec.cl

\section{RESUMEN}

Los insecticidas sintéticos son el método más utilizado para el control de insectos plaga, sin embargo actualmente se ha reportado desarrollo de resistencia y toxicidad para mamíferos. El aceite esencial de especies del género Eucalyptus ha mostrado resultados promisorios en el control de diversos insectos. El objetivo de esta investigación fue evaluar, en laboratorio, la composición química, toxicidad y efecto repelente del aceite esencial de Eucalyptus globulus y Eucalyptus nitens para el control de adultos de Sitophilus zeamais. Los análisis fitoquímicos indicaron que los mayores constituyentes del aceite esencial de ambas especies son 1,8-cineol (eucaliptol) (55,49\% en E. globulus y $59,85 \%$ en E. nitens) y $\alpha$-pineno (18,18\% en E. globulus y $18,36 \%$ en E. nitens). Los aceites de ambas especies no presentaron toxicidad significativa por contacto aunque afectaron la emergencia de la $\mathrm{F}_{1}$ de $S$. zeamais la cual se redujo en más de un $50 \%$ con $E$. globulus en concentraciones de 4 y $8 \%$ sin afectar la germinación de las semillas. En la toxicidad como fumigante se obtuvieron valores de mortalidad superiores al $70 \%$ en los tratamientos de $20,25,30,35 \mu \mathrm{L}$ con E. nitens y con $35 \mu \mathrm{L}$ en E. globulus. Todos los tratamientos fueron repelentes contra $S$. zeamais. Se concluyó que los aceites esenciales de E. globulus y E. nitens presentan propiedades insecticidas significativas como fumigante y repelente para el control de adultos de S. zeamais.

Palabras clave: Terpenos, insecticidas vegetales, efecto fumigante, efecto repelente, gorgojo del maíz.

\section{ABSTRACT}

Synthetic pesticides are the most widely used method to control insect pests. However, today we have insecticide resistance reports and mammal intoxications. The essential oil of Eucalyptus species has shown promising results in the control of several insect pests. The objective of this research was to assess the chemical composition, toxicity and repellent effect under laboratory conditions of essential oil of Eucalyptus globulus and Eucalyptus nitens against adults of Sitophilus zeamais. The phytochemical analysis indicates that the major constituents of both essential oils are 1.8-cineol (eucalyptol) $(55.49 \%$ in E. globulus and $59.85 \%$ in E. nitens) and $\alpha$-pinene $(18.18 \%$ in E. globulus and $18.36 \%$ in E. nitens). The oils of both species showed no significant contact toxicity although they 
affected the $\mathrm{F}_{1}$ emergence of $S$. zeamais, which was reduced in more than $50 \%$ with E. globulus in concentrations of 4 and $8 \%$ without affecting seed germination. Fumigant toxicity values of mortality higher than $70 \%$ were obtained with E. nitens in treatments of 20, 25, 30 and $35 \mu \mathrm{L}$ and in E. globulus with $35 \mu \mathrm{L}$. All treatments were repellents to S. zeamais. We conclude that essential oils of E. globulus and $E$. nitens have significant insecticidal fumigant properties against $S$. zeamais.

Key words: Terpenoids, botanical insecticides, fumigant, repellent effect, maize weevil.

\section{INTRODUCCIÓN}

A nivel mundial, los insectos plaga son los principales agentes dañinos de los cultivos agrícolas. El deterioro, degradación y contaminación de los cereales por los insectos durante el almacenaje o condiciones de campo causa pérdidas económicas significativas, las cuales se transforman en un riesgo para la seguridad alimentaria. Además, tienen un impacto ecológico negativo, ya que se pierden la energía, suelo, agua y recursos no renovables que se utilizan para la producción de granos (FAO, 2009). Estas pérdidas en condiciones de almacenaje pueden asociarse, entre otros agentes, a la presencia de insectos, como Sitophilus zeamais Mutschulsky (Coleoptera: Curculionidae), el cual es considerado mundialmente como una de las principales plagas durante el almacenamiento de cereales como maíz (Zea mays L.), arroz (Oryza sativa L.) y trigo (Triticum aestivum L.), entre otros (Mossi et al., 2011).

El uso de insecticidas sintéticos es una herramienta imprescindible para su control. Sin embargo, ya no están siendo considerados un método eficaz debido a su alta toxicidad y a que su uso irracional ha provocado problemas de desarrollo de resistencia. Igualmente, el uso de estos productos tiene un impacto negativo sobre organismos no blanco como depredadores y parasitoides (Akthar y Isman, 2004). Según Bakkali et al. (2008), los crecientes problemas con los insecticidas sintéticos justifican la necesidad de investigar el desarrollo de nuevas alternativas de control de bajo impacto ambiental, identificando potenciales insecticidas desde fuentes naturales, tales como plantas o microorganismos (Koul et al., 2008; Pant et al., 2014).

En los últimos años, los estudios para el control de plagas de almacén se han centrado en el uso de aceites esenciales, los que constituyen mezclas complejas de compuestos volátiles que pueden contener alrededor de 20 a 60 componentes, los cuales han sido desarrollados por el metabolismo secundario de las plantas como mecanismo de defensa al ataque de herbívoros y patógenos (Miresmailli e Isman, 2014).

Eucalyptus es un género de árboles de la familia Myrtaceae, caracterizado por ser de rápido crecimiento, empleado en plantaciones forestales para la industria papelera y maderera. En Chile, actualmente existen 828.728 hectáreas plantadas con Eucalyptus de las cuales 573.602 ha corresponden a Eucalyptus globulus Labill y 255.126 ha a Eucalyptus nitens H. Deane \& Maiden (INFOR, 2015).

El aceite esencial de Eucalyptus presenta propiedades antisépticas, bactericidas (Mossi et al., 2011) e insecticidas (Koul et al., 2008; Pant et al., 2014). Esto último se debe a la presencia de 1,8-cineol, compuesto característico del genero $\mathrm{Eu}$ calyptus, que ha sido considerado como un fumigante prometedor (Lee et al., 2003). Además, no es tóxico para mamíferos, es de bajo costo, constituye un recurso renovable, posee un manejo que produce abundante follaje como residuo, y es una especie que no está en peligro de conservación.

Estos antecedentes justifican el objetivo de la presente investigación, que consistió en evaluar las propiedades insecticidas e insectistáticas del aceite esencial de E. globulus y E. nitens, a través de la toxicidad por contacto y fumigante y efecto repelente para el control de adultos de S. zeamais, junto con su efecto sobre la germinación del maíz.

\section{MATERIALES Y MÉTODOS}

Los bioensayos se llevaron a cabo en el Laboratorio de Entomología de la Facultad de Agronomía de la Universidad de Concepción, Campus Chillán, Octava Región, Chile.

\section{Material vegetal, insectos y cereal}

La colecta de hojas de Eucalyptus se llevó a cabo en un bosque de 22 años de edad ubicado en la Estación Experimental "El Nogal" (36 $35^{\circ}$ S; $72^{\circ} 4^{\prime} \mathrm{O}$ ), de la Facultad de Agronomía de la Universidad de Concepción Campus Chillán. La identificación de las especies la realizó la botánica de la Facultad de Agronomía, Lic. Angélica Urbina Parra que fue quien originalmente estableció el huerto en 1994. El criterio de colecta fue el de Vogel et al. (1997), que consiste en seleccionar hojas al azar, de los cuatro puntos cardinales y en distintos lugares dentro del árbol. El follaje colectado se lavó con agua potable para eliminar cualquier contaminante, y posteriormente se sometió a destilación por arrastre de vapor por dos horas, en un aparato tipo Clevenger utilizando 
agua destilada. El aceite obtenido se conservó en frio a $4 \pm 1^{\circ} \mathrm{C}$ en envases de vidrio color ámbar con cierre hermético, hasta su utilización en los bioensayos.

Los insectos utilizados en el bioensayo se obtuvieron a partir de colonias que se encuentran de forma permanente en el Laboratorio de Entomología de la Facultad de Agronomía de la Universidad de Concepción. Las colonias se mantuvieron en frascos de vidrio con maíz como sustrato alimenticio en una cámara bioclimática a $30 \pm 1^{\circ} \mathrm{C}$, $60 \pm 5 \%$ humedad relativa (HR) y oscuridad total.

El maíz utilizado en los bioensayos correspondió al cultivar Dekalb DK440 (Anasac Chile S.A) y se obtuvo de la Estación Experimental "El Nogal" dependiente de la Facultad de Agronomía de la Universidad de Concepción. Este se almacenó en un congelador a $-18 \pm 1^{\circ} \mathrm{C}$ para eliminar cualquier infestación y/o contaminación externa por insectos que pudiese alterar los resultados.

\section{Análisis fitoquímico}

Los análisis fitoquímicos de ambos aceites se realizaron por cromatografía de gases en el Laboratorio de Farmacognosia del Departamento de Química y Farmacia perteneciente a la Facultad de Farmacia de la Universidad de Concepción, con el objetivo de identificar los principales constituyentes del aceite esencial de E. globulus y E. nitens.

\section{Bioensayo 1. Toxicidad por contacto con una superficie tratada}

Este bioensayo se llevó a cabo con la metodología de Kouninki et al. (2007); se usaron tubos de ensayo de vidrio de $6 \mathrm{~mL}$ a los que se les aplicó $1 \mathrm{~mL}$ de una solución de acetona con aceite esencial en las concentraciones a evaluar. Luego, en un agitador vortex las soluciones se homogenizaron durante un minuto para permitir que cubriera uniformemente la superficie interior del tubo. En seguida, se eliminó el excedente de solución, y por dos horas a temperatura ambiente se dejó evaporar la acetona. Finalmente, en cada tubo se introdujeron 10 insectos adultos sin diferenciación de sexos de aproximadamente 48 horas de edad, y posteriormente se cubrieron con una tapa perforada cubierta con tela de tul para permitir el intercambio gaseoso y confinar los insectos dentro del tubo durante el bioensayo.

Las concentraciones de aceite esencial evaluadas fueron 0,$25 ; 0,5 ; 1 ; 2 ; 4$ y $8 \%(\mathrm{v} / \mathrm{v})$, más un testigo consistente en solo acetona. Cada tratamiento tuvo 10 repeticiones, las que se almacenaron en una cámara bioclimática a $30 \pm 1^{\circ} \mathrm{C}, 60 \pm 5 \% \mathrm{HR}$ y oscuridad total. La mortalidad se evaluó a las 24 , 48 y 72 horas de iniciado el bioensayo y los resultados obtenidos se corrigieron con la fórmula de Abbott (1925).

\section{Bioensayo 2. Toxicidad por contacto con grano tratado}

Este bioensayo se realizó con la metodología de Obeng-Ofori y Reichmuth (1997), la cual consiste en mezclar, en frascos de vidrio de 0,25 L de capacidad, 100 g de maíz con una solución de aceite esencial, en las concentraciones a evaluar, disueltas en $1 \mathrm{~mL}$ de acetona más un testigo con solo acetona. A continuación los frascos se dejaron por dos horas a temperatura ambiente para que la acetona se evaporara, y posteriormente cada frasco se infestó con 10 parejas de $S$. zeamais adultos de no más de siete días edad. La diferenciación de sexos se realizó con el criterio de Halstead (1963), que señala que el rostrum de la hembra es más largo y menos ornamentado que el del macho. Luego, cada frasco se cubrió con sus tapas perforadas más una tela de tul para permitir el intercambio gaseoso y evitar el escape de insectos. Finalmente, los frascos se almacenaron en la cámara bioclimática en las mismas condiciones señaladas para la cría masiva de insectos.

\section{Variables evaluadas}

Mortalidad de insectos adultos. La mortalidad se evaluó a las 24, 48 y 72 horas y a los 7 y 15 días después de la infestación (DDI) con las 10 parejas de $S$. zeamais. Se cuantificó los insectos vivos y muertos para obtener el porcentaje de mortalidad el que se corrigió con la fórmula de Abbott (1925).

Emergencia de insectos adultos $\left(\mathrm{F}_{1}\right)$. Una vez cumplidos los 15 días de evaluación de la mortalidad se retiraron todos los insectos adultos de cada frasco y éstos solo con el maíz se devolvieron a la cámara bioclimática por 40 días más. Al final de este periodo (55 DDI) se evaluó la emergencia de insectos adultos $\left(\mathrm{F}_{1}\right)$ considerando como $100 \%$ la $\mathrm{F}_{1}$ del testigo.

Pérdida de peso del grano. Se determinó la pérdida de peso del grano a los 55 DDI, por diferencia, entre el peso inicial (100 g) y final del grano. Para corregir una posible diferencia de peso a causa de una pérdida o ganancia de humedad del grano, junto con los tratamientos se almacenaron en la cámara bioclimática cuatro frascos con $100 \mathrm{~g}$ de maíz sin tratar con aceite esencial y sin infestar con insectos, los que también se pesaron al principio y final del bioensayo.

Germinación. A los 55 DDI se realizó una prueba de germinación con 10 semillas por unidad experimental a temperatura ambiente $\left(20 \pm 5^{\circ} \mathrm{C}\right)$, durante siete días en placas Petri con papel filtro humedecido en su base. Los datos de germinación se registraron diariamente durante los siete días 
considerando como $100 \%$ el número de semillas germinadas en el testigo.

\section{Bioensayo 3. Efecto fumigante}

El efecto fumigante se evaluó con la metodología de Brito et al. (2006), que consiste en la aplicación de 10, 15, 20, 25, 30 y $35 \mu \mathrm{L}$ de aceite esencial sobre un papel filtro circular de $5,5 \mathrm{~cm}$ de diámetro adherido interiormente a la tapa de envases plásticos de $150 \mathrm{~mL}$. Cada envase contenía $25 \mathrm{~g}$ de maíz infestado con 10 insectos adultos sin diferenciación de sexo. Se realizaron cuatro repeticiones por tratamiento, las cuales se mantuvieron en una cámara bioclimática en condiciones controladas de $30 \pm 1^{\circ} \mathrm{C}$ y oscuridad total. $\mathrm{La}$ mortalidad se evaluó los 5 DDI y se corrigió con la fórmula de Abbott (1925).

\section{Bioensayo 4. Repelencia}

Este bioensayo se realizó según la metodología de Tavares y Vendramim (2005), que consiste en una arena de libre elección formada por cinco placas Petri plásticas de $5 \mathrm{~cm}$ de diámetro, estando una placa central conectada a las otras cuatro por medio de tubos plásticos de $10 \mathrm{~cm}$ en posición diagonal formando una " $\mathrm{X}$ ". Los tratamientos evaluados fueron 0,$25 ; 0,5 ; 1 ; 2 ; 4$ y $8 \%(\mathrm{v} / \mathrm{v})$ de aceite esencial, en $1 \mathrm{~mL}$ de acetona los cuales se formularon y agitaron en tubos de vidrio cuyo contenido se mezcló con 20 gramos de maíz que se ubicaron en dos placas Petri diagonalmente opuestas. En las otras dos placas se ubicó el testigo consistente en el grano mezclado sólo con acetona. Luego, en el recipiente central se liberaron 20 insectos adultos sin diferenciación de sexos y después de 24 horas se contabilizó el número de insectos por placa. Cada tratamiento tuvo cuatro repeticiones y con los datos obtenidos se calculó el índice de repelencia (IR) de Mazzoneto y Vendramim (2003), donde los valores de IR indican si es un tratamiento atrayente $(I R>1)$, neutro $(I R=1)$ o repelente $(\mathrm{IR}<1)$.

\section{Diseño experimental}

El diseño experimental para todos los bioensayos fue completamente al azar con un arreglo factorial, de 2 × 7 ( 2 especies de Eucalyptus y 6 concentraciones de aceite esencial más un testigo). Los datos se sometieron a un análisis de varianza (ANOVA) y un test de comparación de medias de Tukey, con un nivel de confianza del $95 \%$. En el caso de la germinación de maíz se realizó un análisis no paramétrico de Kruskal Wallis debido a que los datos no cumplieron los supuestos de distribución normal y homogeneidad de la varianza. En todos los análisis se utilizó el programa estadístico Infostat (Balzarini et al., 2008).

\section{RESULTADOS Y DISCUSIÓN}

\section{Análisis fitoquímico}

Los resultados del análisis fitoquímico indican que el mayor constituyente del aceite esencial de E. globulus (Tabla 1) y E. nitens (Tabla 2) es eucaliptol (1,8-cineol) con 55,5 y 59,9\%, respectivamente, coincidiendo con Batish et al. (2008) y Koul et al. (2008), seguido por $\alpha$-pineno en concentraciones de $18,2 \%$ en E. globulus y de $18,4 \%$ en E. nitens.

\section{Mortalidad por contacto con una superficie tratada}

Los resultados obtenidos en este bioensayo indican que solo el aceite esencial de E. globulus puede ser considerado como prometedor según el criterio de Lagunes (1994), al mostrar una mortalidad mayor al $40 \%$ en los tratamientos de 4 y $8 \%$ a las 72 h de iniciado el bioensayo (Tabla 3). Por tanto, se puede inferir que ni E. globulus ni $E$. nitens poseen efecto derribante significativo sobre S. zeamais. Esto coincide parcialmente con Abdelgaleil et al. (2009), quienes evaluaron la toxicidad de monoterpenos aislados obteniendo una baja toxicidad $\left(\mathrm{CL}_{50}>500 \mu \mathrm{g} \mathrm{cm} \mathrm{cm}^{-2}\right)$ con 1,8 cineol (eucaliptol), componente mayoritario de ambos aceites, sobre Sitophilus oryzae L. (Curculionidae) y Tribolium castaneum H. (Tenebrionidae). Por otra parte Tapondjou et al. (2005), con dosis de $1,56 \mu \mathrm{L} \mathrm{cm}^{-2}$ y $0,78 \mu \mathrm{L} \mathrm{cm}^{-2}$ de aceite esencial de Eucalyptus saligna L., y Mossi et al. (2011), con dosis de $0,29 \mu \mathrm{L} \mathrm{cm}{ }^{-2}$ de E. globulus, reportaron una mortalidad por contacto de $100 \%$ con superficie tratada; aunque las dosis evaluadas y metodología utilizada por estos autores no son homologables con la presente investigación, solo se destaca el antecedente de que E. saligna y E. globulus en otros estudios han mostrado toxicidad significativa por contacto con una superficie tratada. La disparidad obtenida con los autores mencionados anteriormente puede deberse a que la concentración de los componentes presentes en los aceites esenciales utilizados por éstos es diferente a la del presente bioensayo, además de que el material utilizado como superficie tratada en esta investigación fueron tubos de vidrio y la adhesión de los compuestos en esta superficie es baja en comparación al papel filtro utilizado por Tapondjou et al. (2005) y Mossi et al. (2011). Además, para evitar la fuga de insectos los tubos se cubrieron con tela de tul y las tapas se perforaron perdiéndose compuestos volátiles que son los que usualmente presentan la mayor actividad insecticida. Cabe señalar también, que la época de recolección del follaje, método de extracción del aceite esencial y órgano utilizado, entre otros, influyen directamente en la concentración de los metabolitos 
Tabla 1. Componentes químicos del aceite esencial de hojas de E. globulus.

Table 1. Chemical constituents of essential oil of leaves of E. globulus.

\begin{tabular}{ccccc}
\hline Peak & RT $^{\mathbf{1}}$ (min) & Composición (\%) & Compuesto & Identificación $^{2}$ \\
\hline 1 & $-5,0$ & 18,18 & 1 R- $\alpha$-pineno & IR, EM \\
2 & $-5,7$ & $-0,69$ & $(-)-\beta$-pineno & IR, EM \\
3 & $-5,9$ & $-0,78$ & Desconocido & IR, EM \\
4 & $-6,5$ & $-0,37$ & o-cimeno & IR, EM \\
5 & $-6,6$ & 55,49 & Eucaliptol & IR, EM, S \\
6 & $-7,0$ & $-0,21$ & Metil m-tolil carbinol & IR, EM \\
7 & $-8,9$ & $-0,33$ & $(-)$-terpinen-4-ol & IR, EM \\
8 & $-9,1$ & $-0,79$ & $\alpha$-terpineol & IR, EM \\
9 & 11,7 & 0,50 & Desconocido & IR, EM \\
10 & 12,2 & $-2,98$ & $\alpha$-gurjuneno & IR, EM \\
11 & 12,5 & $-0,39$ & $(+)$-calereno & IR, EM \\
12 & 12,6 & $-8,15$ & Longiborn-2-eno & IR, EM \\
13 & 12,9 & $-2,09$ & $(-)$-alloaromadendreno & IR, EM \\
14 & 13,3 & $-0,55$ & Desconocido & IR, EM \\
15 & 13,3 & $-3,91$ & (+)-ledeno & IR, EM \\
16 & 13,6 & $-0,37$ & $\alpha$-cadineno & IR, EM \\
17 & 14,1 & $-0,48$ & Desconocido & IR, EM \\
18 & 14,2 & $-0,21$ & Humulano-1,6-dien-3-ol & IR, EM \\
19 & 14,4 & 2,34 & $(-)$-isolongifolol acetato & IR, EM \\
20 & 14,5 & 0,89 & Longifoleno & IR, EM \\
21 & 14.9 & 0,30 & (+)-rosifoliol & IR, EM \\
\hline
\end{tabular}

${ }^{1}$ : RT: Tiempo de retención.

2: Compuestos identificados por comparación con la base de datos del espectro de masa (EM), índice de retención de Kovats (IR) y estándares puros (S).

secundarios presentes y por consecuencia en sus propiedades insecticidas (Vogel et al., 1996; Pérez et al., 2007; Batish et al., 2008). Finalmente según Tapondjou et al. (2005), la susceptibilidad del insecto y su variabilidad genética también influyen en los resultados del ensayo.

\section{Toxicidad por contacto con grano tratado}

En ninguno de los tratamientos, para ambas especies de Eucalyptus, se obtuvo una mortalidad superior al $10 \%$ (Tabla 4 ), por tanto de acuerdo al criterio de Lagunes (1994), los aceites esenciales de E. globulus y E. nitens no pueden ser considerados como prometedores. Nuevamente los resultados difieren con Tapondjou et al. (2005) quienes utilizaron dosis de 75 y $100 \mu \mathrm{L}$ (equivalentes a 0,19 y $0,25 \%$ ) de aceite esencial de $E$. saligna obteniendo un $100 \%$ de mortalidad. La baja toxicidad obtenida en el bioensayo puede deberse a que la concentración de los componentes del aceite esencial de $E$. saligna es diferente a la de $E$. globulus y E. nitens. Según Reyes-Guzmán et al. (2012), el género Eucalyptus no presenta toxicidad por contacto, y por las características de los compuestos volátiles presentes en el aceite esencial, este debería presentar principalmente efecto fu- migante y repelente más que de contacto. Puntualmente Tapondjou et al. (2005) señalaron que la toxicidad de los aceites esenciales de Cupressus sempervirens L. (Cupressaceae) y E. saligna no se debe a la ingestión del grano tratado sino que a otros factores como su efecto fumigante.

Uno de los compuestos con mayor presencia en ambos aceites esenciales es 1,8 cineol (eucaliptol) el cual es característico del género y se le atribuyen las propiedades plaguicidas (Batish et al., 2008). Este compuesto aislado de Artemisia annua L. (Asteraceae) y aplicado tópicamente en el tórax de T. castaneum adultos presentó una dosis letal $50 \%\left(\mathrm{DL}_{50}\right)$ de $108,04 \mu \mathrm{L} \mathrm{mg}^{-1}$ (Thripathi et al., 2001), lo que indica que las propiedades insecticidas de este compuesto pueden estar siendo afectadas por un efecto de antagonismo, probablemente debido a la interacción con otros monoterpenos presentes en el aceite esencial de $E$. globulus o E. nitens. Esto último podría explicarse con lo mencionado por Miresmailli et al. (2006), quienes reportaron que la toxicidad de la mezcla de compuestos presentes en el aceite esencial de Rosmarinus officinalis L. (Lamiaceae) sobre Tetranychus urticae Koch (Acari: Tetranychidae) se ve potenciada o inhibida por compuestos inactivos 
Tabla 2. Componentes químicos del aceite esencial de hojas de E. nitens.

Table 2. Chemical constituents of essential oil of leaves of $E$. nitens.

\begin{tabular}{ccccc}
\hline Peak & RT $^{\mathbf{1}}(\mathbf{m i n})$ & Composición (\%) & Compuesto & Identificación $^{\mathbf{1}}$ \\
\hline 1 & $-5,0$ & 18,36 & $1 \mathrm{R}-\alpha$-pineno & IR, EM \\
2 & $-5,7$ & $-0,59$ & $(-)-\beta$-pineno & IR, EM \\
3 & $-5,9$ & $-0,39$ & Desconocido & IR, EM \\
4 & $-6,1$ & $-0,29$ & Desconocido & IR, EM \\
5 & $-6,5$ & $-0,70$ & o-cimeno & IR, EM \\
6 & $-6,5$ & $-5,66$ & m-menta-6,8-dieno & IR, EM \\
7 & $-6,6$ & 59,85 & Eucaliptol & IR, EM, S \\
8 & $-7,0$ & $-0,28$ & Metil m-tolil carbinol & IR, EM \\
9 & $-8,3$ & $-0,27$ & Sabinil acetato & IR, EM \\
10 & $-8,9$ & $-0,31$ & $(-)$-terpinen-4-ol & IR, EM \\
11 & $-9,1$ & $-0,46$ & $\alpha$-terpineol & IR, EM \\
12 & 11,3 & $-5,01$ & Terpinil acetato & IR, EM \\
13 & 12,2 & $-0,89$ & $\alpha$-gurjuneno & IR, EM \\
14 & 12,5 & $-0,27$ & $(+)$-calereno & IR, EM \\
15 & 12,6 & $-4,05$ & Longiborn-2-eno & IR, EM \\
16 & 12,9 & $-0,96$ & $(-)$-alloaromadendreno & IR, EM \\
17 & 13,3 & $-0,70$ & $(+)-l e d e n o$ & IR, EM \\
18 & 14,4 & $-0,69$ & $(-)$-isolongifolol acetato & IR, EM \\
19 & 14,5 & $-0,26$ & Longifoleno & IR, EM \\
\hline
\end{tabular}

1: RT: Tiempo de retención.

2: Compuestos identificados por comparación con la base de datos del espectro de masa (EM), índice de retención de Kovats (IR) y estándares puros (S).

Tabla 3. Mortalidad a las 24; 48 y 72 horas de Sitophilus zeamais en una superficie de vidrio tratada con aceite esencial de Eucalyptus globulus y Eucalyptus nitens en seis concentraciones.

Table 3. Mortality of Sitophilus zeamais on a glass surface treated with essential oil of Eucalyptus globulus and Eucalyptus nitens in six concentrations at 24, 48 and 72 hours.

\begin{tabular}{lcccc}
\hline \multirow{2}{*}{ Especie } & Concentración (v/v) (\%) & \multicolumn{3}{c}{ Mortalidad (\%) } \\
\cline { 3 - 5 } & & 24 horas & 48 horas & 72 horas \\
\hline E. globulus & 0,25 & 0,0 & 4,7 & 25,9 \\
& 0,50 & 0,0 & 1,8 & 41,7 \\
& 1,00 & 0,0 & 1,8 & 40,5 \\
2,00 & 0,0 & 7,5 & 40,0 \\
& 4,00 & 0,0 & 3,6 & 42,8 \\
E. nitens & 8,00 & 0,0 & 13,3 & 45,2 \\
& 0,25 & 0,0 & 0,0 & 25,0 \\
& 0,50 & 0,0 & 0,0 & 31,7 \\
& 1,00 & 0,0 & 1,8 & 33,6 \\
& 2,00 & 2,0 & 8,7 & 32,1 \\
& 4,00 & 2,0 & 7,7 & 38,3 \\
& 8,00 & 3,0 & 5,6 & 35,2 \\
\hline
\end{tabular}

presentes o ausentes en el propio aceite. Estos mismos autores señalan que en algunos casos su presencia es necesaria para lograr la toxicidad completa del aceite esencial, y agregan que en dos mezclas artificiales una en donde se eliminó 1,8 cineol y otra donde se eliminó $\alpha$-pineno, éstas disminuyeron significativamente la toxicidad en
80 y $84 \%$, respectivamente, lo que indujo a concluir que estos componentes son los principales contribuyentes a la toxicidad del aceite.

Además, en esta investigación se realizó una mezcla artificial de 1,8 cineol, $\alpha$-pineno, $\alpha$-terpineol y bornyl acetato (solo compuestos activos), mostrando resultados de mortalidad más bajos 
Tabla 4. Mortalidad por contacto de Sitophilus zeamais alimentado con maíz tratado con aceite esencial de Eucalyptus globulus y Eucalyptus nitens en seis concentraciones a las 24; 48 y 72 horas y 7 y 15 DDI.

Table 4. Mortality of Sitophilus zeamais by contact toxicity fed with maize treated with essential oil of Eucalyptus globulus and Eucalyptus nitens in six concentrations at 24; 48 and 72 hours and 7 and 15 DDI.

\begin{tabular}{lcccccc}
\hline Especie & Concentración (v/v) (\%) & \multicolumn{7}{c}{ Mortalidad (\%) } \\
\hline & & $24 \mathrm{~h}$ & $48 \mathrm{~h}$ & $72 \mathrm{~h}$ & 7 días & 15 días \\
E. globulus & 0,25 & 0,0 & 0,0 & 0,0 & 0,0 & 0,0 \\
& 0,50 & 0,0 & 0,0 & 0,0 & 0,0 & 0,0 \\
& 1,00 & 0,0 & 1,9 & 3,2 & 3,8 & 7,9 \\
2,00 & 0,0 & 2,8 & 4,1 & 6,4 & 6,6 \\
& 4,00 & 0,0 & 1,9 & 3,8 & 3,8 & 5,6 \\
& 8,00 & 0,0 & 2,8 & 3,8 & 7,7 & 6,6 \\
\hline E. nitens & 0,25 & 0,0 & 0,0 & 0,0 & 0,6 & 0,0 \\
& 0,50 & 1,2 & 0,9 & 0,9 & 1,9 & 0,0 \\
& 1,00 & 0,0 & 0,0 & 0,0 & 2,6 & 2,6 \\
& 2,00 & 0,0 & 0,9 & 0,9 & 2,6 & 2,6 \\
& 4,00 & 0,0 & 0,0 & 0,9 & 3,21 & 3,9 \\
& 8,00 & 1,2 & 3,8 & 6,3 & 6,41 & 5,3 \\
\hline
\end{tabular}

*DDI: días después de la infestación.

que los de la mezcla con todos los componentes. Sin embargo Aguilera et al. (2003) reportaron que, 1,8-cineol pudiese ser el responsable de la actividad insecticida que presenta Eugenia melanadenia Krug \& Urb. (Myrtaceae) en larvas del mosquito Aedes aegypti L. (Culicidadae), indicando que este compuesto se encuentra entre los derivados más tóxicos de las plantas. No obstante en este mismo estudio, Psidium rotundatum Griseb. (Myrtaceae) mostró mayor actividad insecticida, que pudo deberse no solo a 1,8 cineol sino que a la presencia de $\alpha$-pineno que posee un efecto potenciador, amplificando la acción letal de este aceite esencial.

Finalmente, considerando que el protocolo utilizado en este bioensayo incluyó el uso de tapas perforadas para permitir el intercambio gaseoso para evaluar solo la toxicidad por contacto del aceite y evitar el escape de insectos, muchos compuestos volátiles se pierden disminuyendo la toxicidad del aceite. $\mathrm{Al}$ respecto Abdelgaleil et al. (2009) mencionaron que el método del bioensayo puede afectar la toxicidad y eficacia de monoterpenos como 1,8-cineol. Además, la bioactividad del aceite esencial, ya sea insecticida o insectiestático, depende de la composición de éste la cual, como ya se mencionó, varía por diferentes factores además de agentes intrínsecos del insecto (Tapondjou et al., 2005).

\section{Emergencia de insectos adultos $\left(\mathrm{F}_{1}\right)$}

Los tratamientos evaluados de E. globulus y $E$. nitens afectaron la emergencia de insectos adul- tos, obteniéndose los mejores resultados con $E$. globulus, donde la $\mathrm{F}_{1}$ fue de $50,6 \%$ (49,4\% de inhibición) y $47,7 \%$ (52,3\% de inhibición), en las dosis de 4 y $8 \%$, respectivamente (Tabla 5). A pesar de que no se encontraron diferencias estadísticas entre los tratamientos, ni interacción especie-concentración, al analizar las de especies de Eucalyptus por separado, sólo se encontraron diferencias significativas en $E$. nitens entre la concentración de $0,5 \%$ con 4 y $8 \%$ (Tabla 6 ). Por tanto, según el criterio de Páez et al. (1990), E. globulus en la concentración de $8,0 \%$ es el único tratamiento que puede ser considerado como prometedor, ya que reduce la emergencia de la $\mathrm{F}_{1}$ en al menos un $50 \%$ con respecto al testigo.

Sin embargo, estos resultados son inferiores a los obtenidos por Tapondjou et al. (2005), quienes reportaron un $0 \%$ de emergencia de $S$. zeamais con dosis de 75 y $100 \mu \mathrm{L}$ de aceite esencial de E. saligna en $40 \mathrm{~g}$ de maíz (equivalentes a 0,19 y $0,25 \%$ ). Esta diferencia puede deberse a que los componentes del aceite esencial de E. saligna se presentan en concentraciones distintas a las de $E$. globulus y E. nitens. Thripathi et al. (2001) estudiaron el efecto de 1,8-cineol aislado de A. аnnua, en la progenie de $T$. castaneum obteniendo un efecto inhibitorio del $98 \%$ en la eclosión de huevos. La disparidad en la actividad ovicida de 1,8 cineol puede deberse a un efecto antagónico de algún compuesto presente en el aceite esencial de E. globulus o E. nitens, aunque los autores mencionan que los huevos de algunas especies presentan mayor tolerancia a agentes químicos en compa- 
Tabla 5. Emergencia $\left(\mathrm{F}_{1}\right)$ de Sitophilus zeamais adultos, alimentados con maíz tratado con aceite esencial de Eucalyptus globulus y Eucalyptus nitens en seis concentraciones a los 55 DDI.

Table 5. Emergence $\left(\mathrm{F}_{1}\right)$ of Sitophilus zeamais adults, fed with maize treated with essential oil of Eucalyptus globulus and Eucalyptus nitens in six concentrations to 55 DDI.

\begin{tabular}{lcc}
\hline Especie & Concentración (v/v) $\mathbf{( \% )}$ & Emergencia (\%) \\
\hline E. globulus & 0,25 & 80,14 \\
& 0,50 & 82,31 \\
1,00 & 64,98 \\
2,00 & 54,15 \\
4,00 & 50,54 \\
& 8,00 & 47,65 \\
\hline E. nitens & 0,25 & 87,00 \\
& 0,50 & 94,58 \\
& 1,00 & 80,51 \\
& 2,00 & 73,65 \\
& 4,00 & 63,54 \\
8,00 & 62,82 \\
\hline
\end{tabular}

*El análisis de varianza $(\mathrm{P}>0,05)$, test Tukey señala que no existen diferencias estadísticas entre tratamientos. DDI: días después de infestación.

Tabla 6. Emergencia $\left(\mathrm{F}_{1}\right)$ de Sitophilus zeamais adultos, alimentados con maíz tratado con Eucalyptus nitens en seis concentraciones a los 55 DDI.

Table 6. Emergence $\left(\mathrm{F}_{1}\right)$ of Sitophilus zeamais adults, fed with maize treated with Eucalyptus nitens in six concentrations at 55 DDI.

\begin{tabular}{ccc}
\hline E. nitens & Concentración (\%) & Emergencia (\%) \\
\hline 0,25 & $87,00 \mathrm{ab}$ \\
0,50 & $94,58 \mathrm{~b}$ \\
1,00 & $80,51 \mathrm{ab}$ \\
2,00 & $73,65 \mathrm{ab}$ \\
4,00 & $63,54 \mathrm{a}$ \\
8,00 & $62,82 \mathrm{a}$ \\
\hline
\end{tabular}

Tratamientos con la misma letra no son significativamente diferentes. Análisis de varianza $(\mathrm{P}>0,05)$, test Tukey. DDI: días después de infestación.

ración a otros estados de desarrollo del insecto.

La alta emergencia obtenida en este bioensayo se explica principalmente por la baja toxicidad por contacto obtenida en los tratamientos que no afectaron a los adultos o estados inmaduros de $S$. zeamais. Silva-Aguayo et al. (2006) señalan que los menores valores de la $\mathrm{F}_{1}$ se registran con los tratamientos de mayor toxicidad, debido a que las hembras son eliminadas antes de que ovipositen, aunque tampoco debe ser tomada como una regla general, ya que algunas especies vegetales pueden presentar propiedades insectistáticas, provocando una menor oviposición y/o emergencia sin presentar una toxicidad significativa.

\section{Pérdida de peso del grano}

Los resultados obtenidos en este bioensayo muestran una interacción especie-concentración significativa. Sin embargo, esta solo fue significativa para la especie de Eucalyptus y no para la concentración de aceite esencial (Tabla 7). En términos generales, E. globulus resultó ser más efectivo que $E$. nitens, presentando en todos los tratamientos valores más bajos de pérdida de peso, sin observarse diferencias estadísticas entre tratamientos. Usualmente, una mayor mortalidad de insectos relacionada con una menor $\mathrm{F}_{1}$ implica una menor pérdida de peso del grano por lo que estos resultados podrían deberse a la baja mortalidad o posiblemente a un comportamiento de supervivencia por parte de los insectos, ya que éstos al ser expuestos a condiciones adversas o de estrés tienden a alimentarse más, para asegurar su descendencia, produciendo una alta emergencia de la $\mathrm{F}_{1}$ sumada a que el aceite no presentaría efectos disuasivos de la alimentación. Por otro 
Tabla 7. Porcentaje de pérdida de peso de maíz tratado con aceite esencial de Eucalyptus globulus y Eucalyptus nitens, en siete concentraciones a los 55 DDI para el control Sitophilus zeamais.

Table 7. Percentage of weight loss of maize treated with essential oil of Eucalyptus globulus and Eucalyptus nitens, in seven concentrations to 55 DAI at Sitophilus zeamais control.

\begin{tabular}{lcr}
\hline Concentración (v/v) (\%) & \multicolumn{2}{c}{ Pérdida de peso (\%) } \\
& E. globulus & E. nitens \\
\hline 0,0 & $6,19 \mathrm{Aa}$ & $13,73 \mathrm{Ba}$ \\
0,25 & $7,64 \mathrm{Aa}$ & $12,53 \mathrm{Ba}$ \\
0,5 & $7,29 \mathrm{Aa}$ & $12,48 \mathrm{Ba}$ \\
1 & $7,03 \mathrm{Aa}$ & $9,13 \mathrm{Ba}$ \\
2 & $7,07 \mathrm{Aa}$ & $9,12 \mathrm{Ba}$ \\
4 & $6,21 \mathrm{Aa}$ & $12,66 \mathrm{Ba}$ \\
8 & $6,94 \mathrm{Aa}$ & $13,73 \mathrm{Ba}$ \\
\hline
\end{tabular}

Tratamientos con la misma letra mayúscula no son significativamente diferentes entre las especies, y tratamientos con la misma letra minúscula no son significativamente diferentes entre las concentraciones. Análisis de varianza $(P>0,05)$, test Tukey. DDI: días después de infestación.

lado el bioensayo posee factores indirectos, los cuales pueden afectar los resultados, como por ejemplo el uso de semilla no certificada, que presenta calibres diferentes lo cual puede afectar el comportamiento alimenticio del insecto.

\section{Germinación}

En la mayoría de los tratamientos se obtuvo un $100 \%$ de germinación (Tabla 8). En los casos que la germinación no alcanzó la totalidad de las semillas pudo deberse a que no se utilizó semilla certificada con alta variabilidad genética, aunque estos tratamientos no difirieron significativamente del resto. Asimismo, en todas las concentraciones se obtuvo una germinación mayor a $90 \%$, cumpliendo con los requerimientos para que el maíz pueda ser comercializado como semilla (SAG, 2000).

\section{Efecto fumigante}

En este bioensayo se observó una interacción significativa entre la especie del Eucalyptus y la concentración, observándose diferencias estadísticas entre las especies y los tratamientos. La toxicidad obtenida con E. nitens fue mucho mas consistente que la con E. globulus, lo cual es una situación común al utilizar productos naturales que no tiene un proceso de estandarización industrial. Además, esta variabilidad y falta de estandarización han sido uno de los motivos que han hecho que los insecticidas de origen vegetal no se hayan masificado. En general, E. nitens presentó mayor toxicidad que E. globulus, con una mortalidad superior al $40 \%$ a partir de los $15 \mu \mathrm{L}$; en cambio E. globulus sólo alcanzó una toxicidad confiable a los $35 \mu \mathrm{L}$ con $82,1 \%$ (Tabla 9).

A pesar de que ambas especies presentan tratamientos promisorios, según el criterio de Lagu- nes (1994), los resultados no concuerdan con Cárdenas et al. (2013) y Lamiri et al. (2001), quienes obtuvieron baja toxicidad como fumigante de $E$. globulus contra $A$. aegypti y $S$. zeamais, respectivamente. Al mismo tiempo estos resultados difieren de los de Reyes-Guzmán et al. (2012), quienes reportaron un $100 \%$ de mortalidad de Rhyzopertha dominica Fabricius (Bostrychidae) en dosis de 10 y $15 \mu \mathrm{L}$ con aceite esencial de E. globulus, lo que demuestra la alta variabilidad en la efectividad biológica de los aceites esenciales.

Sin embargo, los resultados del presente estudio concuerdan con Mediouni Ben Jemâa et al. (2013), quienes con aceite esencial de E. camaldulensis y E. leucoxylon obtuvieron sobre un $90 \%$ de mortalidad en larvas y adultos de Ectomyelois ceratoniae Zeller (Pyralidae), resultando más efectivo E. camaldulensis que E. leucoxylon. Los resultados también coinciden con Lee et al. (2001), quienes reportaron una significativa actividad fumigante del aceite esencial sobre $S$. oryzae de una especie no identificada del genero Eucalyptus, con una concentración de $81,1 \%$ de 1,8-cineol.

Igualmente Abdelgaleil et al. (2009) evaluaron la toxicidad como fumigante de diferentes monoterpenos sobre T. castaneum y S. oryzae, encontrando que 1,8-cineol tuvo la mayor toxicidad sobre ambas especies, con valores de $\mathrm{CL}_{50}$ de $17,16 \mathrm{y}$ $14,19 \mathrm{mg} \mathrm{L}^{-1}$, respectivamente. Además, Lee et al. (2004) señalaron a 1,8-cineol como el responsable de la toxicidad como fumigante del aceite esencial de Eucalyptus blakelyi M. (Myrtaceae) y Melaleuca fulgens R.Br. (Myrtaceae), aunque también destacan a otros constituyentes del grupo de los monoterpenos que se encuentran en concentraciones menores como $\alpha$-pineno que pueden potenciar el efecto insecticida de 1,8-cineol, coincidiendo con Ebadollahi y Mahboubi (2011), que reportaron un 
Tabla 8. Germinación del maíz tratado aceite esencial de Eucalyptus globulus y Eucalyptus nitens, en siete dosis.

Table 8. Germination of maize treated with essential oil of Eucalyptus globulus and Eucalyptus nitens, in seven concentrations.

\begin{tabular}{lcc}
\hline Especie & Concentración (v/v) (\%) & Germinación (\%) \\
\hline E. globulus & 0,00 & $100,0 \mathrm{a}$ \\
& 0,25 & $095,0 \mathrm{a}$ \\
0,50 & $100,0 \mathrm{a}$ \\
1,00 & $100,0 \mathrm{a}$ \\
2,00 & $100,0 \mathrm{a}$ \\
4,00 & $100,0 \mathrm{a}$ \\
& 8,00 & $097,5 \mathrm{a}$ \\
\hline E. nitens & 0,00 & $100,0 \mathrm{a}$ \\
& 0,25 & $097,5 \mathrm{a}$ \\
& 0,50 & $100,0 \mathrm{a}$ \\
& 1,00 & $100,0 \mathrm{a}$ \\
& 2,00 & $100,0 \mathrm{a}$ \\
4,00 & $100,0 \mathrm{a}$ \\
8,00 & $100,0 \mathrm{a}$
\end{tabular}

*Tratamientos con la misma letra no son significativamente diferentes. Prueba de Kruskal Wallis $(\mathrm{P}>0,05)$.

Tabla 9. Efecto fumigante a los 5 DDI de aceite esencial de Eucalyptus globulus y Eucalyptus nitens en seis dosis sobre Sitophilus zeamais.

Table 9. Fumigant effect against Sitophilus zeamais of essential oil of Eucalyptus globulus and Eucalyptus nitens in six concentrations at 5 DDI.

\begin{tabular}{ccc}
\hline Dosis $(\mu \mathrm{L})$ & \multicolumn{2}{c}{ Mortalidad (\%) } \\
\cline { 2 - 3 } 10 & E. globulus & E. nitens \\
15 & $30,8 \mathrm{Aab}$ & $26,9 \mathrm{Ab}-$ \\
20 & $03,8 \mathrm{Ab}_{-}$ & $56,4 \mathrm{Bab}$ \\
25 & $05,8 \mathrm{Ab}_{-}$ & $79,5 \mathrm{Bab}$ \\
30 & $48,7 \mathrm{Aab}$ & $94,9 \mathrm{Ba}-$ \\
35 & $30,8 \mathrm{Aab}$ & $82,1 \mathrm{Bab}$ \\
\hline
\end{tabular}

* Tratamientos con la misma letra mayúscula no son significativamente diferentes entre las especies, y tratamientos con la misma letra minúscula no son significativamente diferentes entre las dosis. Análisis de varianza $(P>0,05)$, test Tukey. DDI: días después de infestación.

$100 \%$ de mortalidad de T. castaneum y S. granarius con aceite esencial de Azilia eryngioides Hedge \& Lamond (Apiaceae), que tiene como componente mayoritario $(63,8 \%)$ a $\alpha$-pineno.

\section{Efecto repelente}

Todos los tratamientos evaluados de E. globulus o E. nitens fueron repelentes, ya que en todas las concentraciones evaluadas el índice de repelencia (IR) mostró valores menores a 1 (Mazzoneto y Vendramim, 2003) (Tabla 10). Estos resultados coinciden con Mishra et al. (2012), quienes señalan que $E$. globulus posee actividad repelente debido a la presencia de 1,8 cineol, que podría actuar tanto solo como sinérgicamente con los demás compuestos del aceite esencial. Además estos mismos autores señalan que a pesar de que el modo de acción del aceite esencial aún no se ha identificado completamente, la repelencia de los insectos puede deberse a la asfixia e inhibición de diferentes procesos de su metabolismo. Por otro lado Mossi et al. (2011), también encontraron efecto repelente contra $S$. zeamais en aceites esenciales de especies de la familia Myrtaceae, como E. globulus, E. saligna y E. dunnii, entre otros, mencionando que no existe una correlación entre la concentración de 1,8 cineol y $\alpha$-pineno y el efecto repelente, lo que indica que estos compuestos 
Tabla 10. Repelencia de adultos de Sitophilus zeamais alimentados con maíz tratado con aceite esencial de Eucalyptus globulus y Eucalyptus nitens en seis concentraciones.

Table 10. Repellency of adults of Sitophilus zeamais fed with maize treated with essential oil of Eucalyptus globulus and Eucalyptus nitens in six concentrations.

\begin{tabular}{lccl}
\hline Especie & Concentración $(\mathbf{v} / \mathbf{v}) \mathbf{( \% )}$ & Índice de repelencia (IR) $^{\mathbf{1}}$ & Efecto $^{\text {E. globulus }}$ \\
\hline 0,25 & 0,62 & Repelente \\
& 0,50 & 0,52 & Repelente \\
1,00 & 0,55 & Repelente \\
2,00 & 0,55 & Repelente \\
& 4,00 & 0,53 & Repelente \\
& 8,00 & 0,43 & Repelente \\
\hline E. nitens & 0,25 & 0,68 & Repelente \\
& 0,50 & 0,47 & Repelente \\
& 1,00 & 0,39 & Repelente \\
& 2,00 & 0,57 & Repelente \\
& 4,00 & 0,45 & Repelente \\
& 8,00 & 0,36 & Repelente \\
\hline
\end{tabular}

IIR: Índice de Repelencia: IR > 1 atrayente; IR = 1 neutro; IR $<1$ repelente.

no actúan de forma aislada sino que en conjunto con otros presentes en el aceite esencial, como citronelal, citronelol, citronelil de etilo, p-cimeno, eucamalol, limoneno, linalool, $\alpha$-pineno, $\gamma$-terpinene, $\alpha$-terpineol, alloocimene y aromadendrene.

A partir de los resultados obtenidos se puede inferir que el aceite esencial de E. globulus y E. nitens, presentan efecto insectistático e insecticida y por tanto tienen potencial para actuar como estrategia preventiva (repelente) y curativa (fumigante) para evitar posibles infestaciones por insectos en el cereal durante el almacenamiento.

\section{CONCLUSIONES}

De los resultados obtenidos en el presente estudio se puede concluir que el aceite esencial de E. globulus y E. nitens no presentan toxicidad por contacto pero si toxicidad como fumigante y repelente sobre S. zeamais, sin afectar significativamente la germinación del maíz.

\section{LITERATURA CITADA}

Abbott, W.S. 1925. A method of computing the effectiveness of an insecticide. J. Econ. Entomol. 18(2):265-267.

Abdelgaleil, S.A.M., M.I.E. Mohamed, M.E.I. Badawy, and S.A.A. El-Arami. 2009. Fumigant and contact toxicities of monoterpenes to Sitophilus oryzae (L.) and Tribolium castaneum (Herbst) and their inhibitory effects on acetylcholinesterase activity. J. Chem. Ecol. 35(5):518-525.
Aguilera, L., A. Navarro, J.E. Tacoronte, M. Leyva, y M.C. Marquetti. 2003. Efecto letal de myrtaceas cubanas sobre Aedes aegypti (Diptera: Culicidae). Rev. Cuba. Med. Trop. 55(2):100104.

Akhtar, Y., and M.B Isman. 2004. Comparative growth inhibitory and antifeedant effects of plant extracts and pure allelochemicals on four phytophagous insect species. J. Appl. Entomol. 128(1):32-38.

Bakkali, F., S. Averbeck, D. Averbeck, and M. Idaomar. 2008. Biological effects of essential oils. A review. Food Chem. Toxicol. 46(2):446-475.

Balzarini, M.G., L. González, M. Tablada, F. Casanoves, J.A. Di Rienzo, y C.W. Robledo. 2008. InfoStat: software estadístico. Manual del usuario. Versión 2008. Brujas. Córdoba, Argentina.

Batish, D.R., H.P. Singh, R.K. Kohli, and S. Kaur. 2008. Eucalyptus essential oil as a natural pesticide. For. Ecol. Manag. 256(12):2166-2174.

Brito, J.P., J.E. Oliveira, e S.A. De Bortoli. 2006. Toxicidade de óleos essenciais de Eucalyptus spp. sobre Callosobruchus maculatus (Fabr., 1775) (Coleoptera: Bruchidae). Rev. Biol. Ciênc. Terra 6(1):96-103.

Cárdenas, E., I. Riveros, y L. Lugo. 2013. Efecto insecticida de cuatro aceites esenciales sobre adultos de Aedes aegypti y Anopheles albimanus en condiciones experimentales. Entomotropica 28(1):1-10. 
Ebadollahi, A., and M. Mahboubi. 2011. Insecticidal activity of the essential oil isolated from Azilia eryngioides (Pau) Hedge et Lamond against two beetle pests. Chilean J. Agric. Res. 71(3):406-411.

FAO. 2009. Post-harvest losses aggravate hunger. Food and Agriculture Organization of the United Nations (FAO), Italy. Available at http://www.fao.org/news/story/en/ item/36844/icode/. (Accessed 01 October 2015).

Halstead, D.G.H. 1963. External sex differences in stored-products Coleoptera. Bull. Entomol. Res. 54(1):119-134.

INFOR. 2015. El sector forestal en Chile. 50 p. Instituto Forestal (INFOR), Santiago, Chile.

Koul, O., S. Walia, and G.S. Dhaliwal. 2008. Essential oils as green pesticides: potential and constraints. Biopes. Int. 4(1):63-84.

Kouninki, H., T. Hance, F.A. Noudjou, G. Lognay, F. Malaisse, M.B. Ngassoum, et al. 2007. Toxicity of some terpenoids of essential oils of Xylopia aethiopica from Cameroon against Sitophilus zeamais Motschulsky. J. Appl. Entomol. 131(4):269-274.

Lagunes, A. 1994. Extractos y polvos vegetales, y polvos minerales para el combate de plagas del maíz y del frijol en la agricultura de subsistencia. Memoria. Colegio de Postgraduados en Ciencias Agrícolas, Texcoco, México.

Lamiri, A., S. Lhaloui, B. Benjilali, and M. Berrada. 2001. Insecticidal effects of essencial oils against Hessian fly, Mayetiola destructor (Say). Field Crops Res. 71(1):9-15.

Lee, B.-H., W.-S. Choi, S.-E. Lee, and B.-S. Park. 2001. Fumigant toxicity of essential oils and their constituent compounds towards the rice weevil, Sitophilus oryzae (L.). Crop Prot. 20(4):317-320.

Lee, B.-H., P.C. Annis, and F. Tumaalii. 2003. The potential of 1,8-cineole as a fumigant for stored wheat. In E.J. Wright, M.C. Webb and E. Highley (eds.). Proceedings of the Australian postharvest technical conference. June 25-27, 2003. Commonwealth Scientific and Industrial Research Organisation, Stored Grain Research Laboratory, Canberra, Australia.

Lee, B.-H., P.C. Annis, F. Tumaalii, and S.-E. Lee. 2004. Fumigant toxicity of Eucalyptus blakelyi and Melaleuca fulgens essential oils and 1,8-cineole against different development stages of the rice weevil Sitophilus oryzae. Phytoparasitica 32(5):498-506.

Mazzonetto, F., e J.D. Vendramim. 2003. Efeito de pós de origem vegetal sobre Acanthoscelides obtectus (Say) (Coleoptera: Bruchidae) em feijão armazenado. Neotrop. Entomol. 32(1):145-149.
Mediouni Ben Jemâa, J., S. Haouel, and M.L. Khouja. 2013. Efficacy of eucalyptus essential oils fumigant control against Ectomyelois ceratoniae (Lepidoptera: Pyralidae) under various space occupation conditions. J. Stored Prod. Res. 53:67-71.

Miresmailli, S., R. Bradbury, and M.B. Isman. 2006. Comparative toxicity of Rosmarinus officinalis L. essential oil and blends of its major constituents against Tetranychus urticae Koch (Acari: Tetranychidae) on two different host plants. Pest Manag. Sci. 62(4):366-371.

Miresmailli, S., and M.B. Isman. 2014. Botanical insecticides inspired by plant-herbivore chemical interactions. Trends Plant Sci. 19(1):29-35.

Mishra, B.B., S.P. Tripathi, and C.P.M. Tripathi. 2012. Repellent effect of leaves essential oils from Eucalyptus globulus (Mirtaceae) and Ocimum basilicum (Lamiaceae) against two major stored grain insect pests of Coleopterons. Nat. Sci. 10(2):50-54.

Mossi A.J., V. Astolfi, G. Kubiak, L. Lerin, C. Zanella, G. Toniazzo, et al. 2011. Insecticidal and repellency activity of essential oil of Eucalyptus sp. against Sitophilus zeamais Motschulsky (Coleoptera, Curculionidae). J. Sci. Food Agric. 91(2):273-277.

Obeng-Ofori, D., and C. Reichmuth. 1997. Bioactivity of eugenol, a major component of essential oil of Ocimum suave (Wild.) against four species of stored-product Coleoptera. Int. J. Pest Manag. 43(1):89-94.

Páez, A., A. Lagunes, J.L. Carrillo, y J.C. Rodríguez. 1990. Polvos vegetales y materiales inertes para el combate del gorgojo Sitophilus zeamais (Coleoptera: Curculionidae) en maíz almacenado. Agrociencia Serie Protección Vegetal 1(3):35-46.

Pant, M., S. Dubey, P.K. Patanjali, S.K. Naik, and S. Sharma. 2014. Insecticidal activity of eucalyptus oil nanoemulsion with karanja and jatropha aqueous filtrates. Int. Biodeterior. Biodegran. 91:119-127.

Pérez, F., G. Silva, M. Tapia, y R. Hepp. 2007. Variación anual de las propiedades insecticidas de Peumus boldus sobre Sitophilus zeamais. Pesq. Agropec. Bras. 42(5):633-639.

Reyes-Guzmán, R., J. Borboa-Flores, F.J. Cinco-Moroyoqui, E.C. Rosas-Burgos, P.S. Osuna-Amarillas, F.J. Wong-Corral, et al. 2012. Actividad insecticida de aceites esenciales de dos especies de Eucalyptus sobre Rhyzopertha dominica y su efecto en enzimas digestivas de progenies. Rev. Chapingo Ser. Cienc. For. Ambient. 18(3):385-394. 
SAG. 2000. Normas generales y específicas de certificación de semillas. Servicio Agrícola y Ganadero (SAG), Chile. Disponible en http:// www.sag.gob.cl/sites/default/files/NORMAS_GENERALES_SEMILLAS.pdf (Consulta 21 septiembre 2015).

Silva-Aguayo, G., R. Hepp-Gallo, M. Tapia-Vargas, P. Casals-Bustos, G. Bustos-Figueroa, y F. Osses-Ruiz. 2006. Evaluación de boldo (Peumus boldus Molina) y cal para el control de Sitophilus zeamais Motschulsky. Agrociencia 40(2):219-228.

Tapondjou, A.L., C. Adler, D.A. Fontem, H. Bouda, and C. Reichmuth. 2005. Bioactivities of cymol and essential oils of Cupressus sempervirens and Eucalyptus saligna against Sitophilus zeamais Motschulsky and Tribolium confusum du Val. J. Stored Prod. Res. 41(1):91-102.

Tavares, M.A.G.C., e J.D. Vendramim. 2005. Bioatividade da Erva-de-Santa-Maria, Chenopodium ambrosioides L., sobre Sitophilus zeamais Mots. (Coleoptera: Curculionidae). Neotrop. Entomol. 34(2):319-323.
Tripathi, A.K, V. Prajapati, K.K. Aggarwal, and S. Kumar. 2001. Toxicity, feeding deterrence, and effect of activity of 1,8-cineole from Artemisia annua on progeny production of Tribolium castanaeum (Coleoptera: Tenebrionidae). J. Econ. Entomol. 94(4):979-983.

Vogel, H., J. Muñoz, y I. Razmilic. 1996. Efecto de la época de cosecha sobre el rendimiento de productos deshidratados y aceite esencial en orégano (Origanum vulgare L.). Cienc. Investig. Agrar. 23(2-3):55-60.

Vogel, H., I. Razmilic, y U. Doll. 1997. Contenido de aceite esencial y alcaloides en diferentes poblaciones de boldo (Peumus boldus Mol.). Cienc. Investig. Agrar. 24(1):1-6. 